\title{
Ein Klassifizierungssystem für Industrielle Augmented Reality Anwendungen
}

Jan Luca Siewert, Matthias Neges, Detlef Gerhard

Mit dem Fortschreiten der Digitalisierung in der Industrie findet sich Augmented Reality $(A R)$ in immer mehr Einsatzbereichen. Dennoch bleibt die industrielle Verbreitung trotz sich stetig entwickelnder Technik hinter den Prognosen zurück. Es existieren bereits Arbeiten, die sich mit der Klassifizierung von AR jedoch mit Fokus auf die tatsächliche Implementierung bzw. Umsetzung der Anwendung beschäftigen. Um Anwendungsgebiete und damit die eigentliche Problemstellung, in denen AR einen Mehrwert bieten kann, besser vergleichen und Anforderungen für industrielle Bereiche ableiten zu können, stellt dieser Beitrag ein Klassifizierungssystem für diese Einsatzgebiete vor. Auf vorhergehenden Arbeiten aufbauend wird gezeigt, dass eine Klassifizierung der Einsatzszenarien auf Basis der drei Dimensionen zu unterstützende Aktion, Lebenszyklusphase und Grad der Hilfestellung erfolgen kann.

Dazu wird eine systematische Literaturrecherche von industriellen AR Anwendungen und Studien der Jahre 2016 bis 2020 durchgeführt und nach dem vorgeschlagenen Schema klassifiziert. Neben den daraus gewonnen Erkenntnissen werden in den Beiträgen verwendete Technologien, wie die Darstellungstechnik, der Detailierungsgrad, der Reifegrad der Anwendung und die Art der Inhaltserstellung analysiert. Außerdem werden Probleme bei der Umsetzung sowie künftige Forschungsthemen und -schwerpunkte herausgearbeitet.

Keywords: Augmented Reality, Industrie 4.0, Studie, AR Anwendungen

\section{Einleitung}

Als Augmented Reality (AR) wird die Erweiterung der Realität mit virtuellen Inhalten verstanden. Azuma (1997) charakterisiert eine AR-Anwendung mithilfe drei erforderlicher Kriterien: Die Anwendung muss Reales und Virtuelles kombinieren, in Echtzeit interaktiv sein und an einem festen Platz verortet, sprich in 3D registriert sein. Azuma fordert explizit keine spezielle Darstellungstechnik, wie ein Head-Up-Display. Auch Monitor- Projektions- und Tablet-basierte Lösungen können die Definition von AR erfüllen. Im Umkehrschluss kann nicht jede Lösung mit einem Head-Up-Display als AR bezeichnet werden.

Bereits die ersten Beispiele für AR haben einen industriellen Einsatz im Fokus (Caudell \& Mizell, 1992). Auch Azuma nennt die Industrie, und dabei besonders die Montage, 
als eine zentrale Anwendung für AR. Seitdem sind eine Vielzahl von Prototypen vorgestellt worden, in denen industrielles Augmented Reality eingesetzt wird. Neben der Montage sind Wartung und Service, die Inbetriebnahme, aber auch das Training mögliche Einsatzgebiete.

Obwohl die ersten Prototypen für industrielle AR bereits drei Jahrzehnte zurückliegen, befinden sich nach wie vor nur wenige Systeme im produktiven Einsatz. Um die Gründe dafür besser beschreiben zu können müssen existierende Prototypen besser vergleichbar werden. Es existieren bereits eine Reihe Klassifizierungen, die sich aber häufig auf die tatsächliche, technische Umsetzung von AR Anwendungen beziehen. Um Nutzungspotentiale, gemeinsame Anforderungen und aktuelle Probleme in technischen Umsetzungen besser beschreiben zu können, ist aber eine Klassifizierung der möglichen Einsatzgebiete in der Industrie nötig.

Dieser Beitrag stellt zunächst den aktuellen Stand der Forschung bezüglich aktueller Klassifizierungsformen kurz da. Daraus wird eine Klassifizierung abgeleitet, die sich ausschließlich auf das Einsatzgebiet in der Industrie bezieht. Um dies zu validieren, wird eine systematische Literaturrecherche durchgeführt. Die so identifizierten Umsetzungen werden nach dem erarbeiteten System klassifiziert. Zum Abschluss werden kurz häufige Probleme zusammengefasst und ein Ausblick auf zukünftige Forschungsthemen gegeben.

\section{Klassifizierungssystem für industrielle AR-Anwendungsgebiete}

In der Literatur finden sich verschiedene Versuche, AR und AR Anwendungsgebiete zu klassifizieren. Dabei beziehen sich die wenigsten explizit auf industrielle Anwendungen, sondern versuchen, AR allgemein einzuordnen.

Verschiedene Autoren geben einen Überblick über Stand der Forschung für IAR Anwendungen. Del Fernández Amo, Erkoyuncu, Roy, Palmarini und Onoufriou (2018) untersuchen AR für Instandhaltungsprozesse unter den Aspekten Inhaltserstellung, "Context-Awareness", also die Anpassungsfähigkeit des Systems in Bezug auf den Anwender und die Aufgabe, und die Form der Interaktion. Fraga-Lamas, FernandezCarames, Blanco-Novoa und Vilar-Montesinos (2018) untersuchen AR Anwendung im Schiffsbau. Bottani und Vignali (2019) geben einen Überblick über die historische Entwicklung von industrieller AR in den Jahren 2006 bis 2017, gehen dabei aber nur oberflächlich auf die verschiedenen Einsatzmöglichkeiten ein. Souza Cardoso, Mariano, Flávia Cristina Martins Queiroz und Zorzal (2019) beschreiben industrielle AR im Zeit- 
raum 2012-2018, untersuchen die Ergebnisse aber vor allem in Hinblick auf die Implementierung, wie die verwendete Darstellungs- oder Trackingart. Bezogen auf einen ähnlichen Zeitraum (2011 - 2018) sammeln Egger und Masood (2020) Anwendungsstudien für industrielle AR Anwendungen und betrachten das Anwendungsgebiet, die Darstellungs- und Markerform, sowie die Art der Studie und verwendete Gütekriterien der Anwendungen.

Um industrielle AR Anwendungen zu klassifizieren existieren verschiedene Ansätze. Normand, Servières und Moreau (2012) entwickeln eine Taxonomie für AR Implementierung. Dazu nutzen Sie die vier Dimensionen der Anzahl der Freiheitsgrade des Trackings, den Umfang der Erweiterung, den zeitlichen Zusammenhang zwischen Anwendung und Inhalten und den Detailierungsgrad der Darstellung. Brockmann, Krueger, Stieglitz und Bohlsen (2013) klassifizieren kollaborative Anwendungen nach räumlicher Verteilung der Anwender, zwischen synchroner und asynchroner Bearbeitung, nach Beweglichkeit der Anwender. Zusätzlich differenziert er zwischen Anwendungen mit Avataren oder virtuellen Objekten, nach der Anzahl der Rollen im System sowie nach verwendeter Darstellungstechnik. Wang, Kim, Love und Kang (2013) unterscheiden nach Detailierungsgrad, der Rechen- und Interaktionseinheit, zwischen OpticalSee-Through und Video-See-Through Anwendung sowie nach verwendetem Display und Markertyp. Allen Klassifizierungen ist gemein, dass sie ausschließlich die Implementierung beschreiben, nicht mögliche Anwendungsfälle. Außerdem beschränkt sich keine auf industrielle Systeme und deren Eigenheiten.

Fite-Georgel (2011) beschreibt mögliche industrielle Anwendungsgebiete ausschließlich nach deren Auftreten im Produktlebenszyklus. Dabei stützt er sich auf die Phasen nach Ludwig (2015) Produktdesign, Montage, Inbetriebnahme, Wartung und Service sowie Entsorgung. Röltgen und Dumitrescu (2020) erweitern dieses Schema. Sie unterscheiden je vier kontextuelle und technologische Dimensionen. Das Anwendungsgebiet wird nach den oben genannten Lebenszyklusphasen, nach der zu unterstützenden Aktion nach Ludwig (2015) (Informieren, Planen, Ausführen, Kontrollieren), nach der zeitlichen und räumlichen Art der Zusammenarbeit und ob die reale oder eine virtuelle Umgebung vom Anwender manipuliert wird unterschieden. Neben diesen kontextuellen Dimensionen differenzieren Röltgen und Dumitrescu nach dem räumlichen und zeitlichen Bezug und der angestrebten realitätsnähe der Darstellung. Die letzte Dimension unterscheidet die Zielstellung. AR Anwendungen können zu einer Leistungssteigerung, Qualifikation oder einer besseren Wahrnehmung beitragen. Neben diesen acht Dimensionen leiten Röltgen und Dumitrescu 16 industrielle Anwendungsbereiche aus einer systematischen Literaturrecherche ab. 


\section{Methodik}

Während vorangegangen Arbeit die Klassifizierungskriterien in der Regel induktiv aus den gesammelten Quellen ableiten, soll in diesem Beitrag ein deduktives Klassifizierungsschema für industrielle Anwendungsgebiete vorgestellt werden, das auf den vorgestellten Stand der Technik aufbaut.

Analog zu Röltgen und Dumitrescu ist die auszuführende Aktion des Assistenzsystems nach Ludwig unterteilt in

- Ausführen,

- Planen,

- Informieren und

- Kontrollieren.

Die zweite Dimension ist, wie in Fite-Georgel (2011) und Röltgen und Dumitrescu, die Phase im Produktlebenszyklus, in der der zu unterstützende Prozess fällt. Um Aufgaben wie z.B. in der Verfahrenstechnik besser einordnen zu können wird die dort verwendete Kategorie Inbetriebnahme zur Phase Montage erweitert und von der vorangehenden Produktion abgegrenzt. Außerdem wird zusätzlich die Phase des Betriebs berücksichtigt, in der z.B. Anwendungen in der Logistik zugeordnet werden können. Die sechs Ausprägung der Dimension Lebenszyklusphase sind daher abweichend von Grieves (2006)

- Produktentwicklung,

- Produktion,

- Montage,

- Betrieb,

- Wartung und Service sowie

- Entsorgung.

Die dritte Dimension ist der Grad der Unterstützung, der Einfluss auf die Zielgruppe des Systems hat. Obwohl die Relevanz dieses Aspekts bei der Gestaltung von AR Systemen häufig betont wird (Erkoyuncu, Del Amo, Dalle Mura, Roy \& Dini, 2017; Gattullo et al., 2020), wird er die Klassifizierungen des Anwendungsgebiets bisher nicht berücksichtigt. So muss ein Facharbeiter anders angeleitet werden als eine Person in der Ausbildung oder eine ungelernte Arbeitskraft. Menschen mit kognitiven Einschränkungen 
benötigen etwa eine noch größere, durchgehende Hilfestellung durch AR. In diesem Beitrag wird zwischen einem geringen, einem mittleren und einem hohen Grad der Hilfestellung unterschieden. Die Zielgruppe selber wird nicht explizit verwendet, da sich deren Anforderungen während des Einsatzes ändern können und verschiedene Zielgruppen einen ähnlichen Unterstützungsgrad erfordern.

Um diese deduzierte Klassifikation zu validieren, wird eine systematische Literaturrecherche durchgeführt. Da sich die technologischen Möglichkeiten von AR laufend erweitern, soll mit dem Betrachtungszeitraum 2016 bis 2020 auch neuere Anwendungen berücksichtigt werden als in den vorgestellten, vorangegangenen Beiträgen. Das Vorgehen folgt dabei dem Systematic Review nach Tranfield, Denyer und Smart (2003). Zunächst wird die Fragestellung klar definiert. Im Anschluss werden relevante Datenbanken identifiziert und konkrete Suchbegriffe erarbeitet. Nach einer Filterung von Duplikaten werden nicht-relevante Ergebnisse anhand klarer Kriterien zunächst auf Basis des Titels und des Abstracts ausgeschlossen. Die verbleibenden Publikationen werden dann im Volltext analysiert. Die Publikationen werden in die vorgeschlagenen Kategorien sortiert. Zum Abschluss werden genannte Probleme mit der Technologie herausgearbeitet.

Ziel der systematischen Recherche ist die Identifikation aller publizierten Prototypen von AR im industriellen Umfeld. Um die Ergebnisse einzuschränken und die Überschneidung mit vorangegangen Literatur Reviews gering zu halten, beschränkt sich dieser Beitrag auf Publikationen aus den Jahren 2016 bis 2020 auf Deutsch oder Englisch. Neben der Validierung des Klassifizierungssystems sollen mit einer einfachen Inhaltsanalyse Probleme zusammengefasst werden.

Im zweiten Schritt wurden die Datenbanken Web Of Science, Scopus und IEEE Explore für Englischsprachige und TEMA für Deutschsprachige Beiträge ausgewählt. Der allgemeine Suchbegriff "Augmented Reality“ wurde dabei zunächst mit der Anforderung kombiniert, industrielle Anwendungen zu beschreiben. Daher wurde, neben dem allgemeinen "Industrie“, auch die Anwendungsgebiete (Fite-Georgel, 2011) eingeschlossen. Da ausschließlich konkrete Umsetzungen untersucht werden, wird noch das Schlüsselwort "Anwendung" , "System" oder "Studie" ergänzt. Alle Suchbegriffe können sowohl in Deutscher als auch in Englischer Sprache im Titel vorkommen. Die konkreten Suchbegriffe nach Datenbank sowie die Trefferzahl zeigt Tabelle 1. Nach dem Filtern von Duplikaten basierend auf der DOI sind 283 konkrete Publikationen identifiziert worden. 
Tabelle 1: Verwendete Datenbanken Suchbegriffe und Trefferzahlen

\begin{tabular}{|c|c|c|}
\hline Datenbank & Suchanfrage & Trefferzahl \\
\hline Web Of Science & $\begin{array}{l}\text { TI=(("Augment* Realit*" OR "AR" OR "Erweitert* Realität") AND (In- } \\
\text { dustr* OR Mangement OR Service OR Maintenance OR Logistic OR } \\
\text { Quality OR Instandhaltung OR Wartung OR Logistik OR Education } \\
\text { OR Schulung) AND (Study OR Studie OR "Use-Case" or Application } \\
\text { or Anwendung or System) ) }\end{array}$ & 186 \\
\hline Scopus & $\begin{array}{l}\text { TITLE ( ( "Augment* Realit*" OR "AR" OR "Erweitert* Realität" ) } \\
\text { AND ( industr* OR mangement OR service OR maintenance OR } \\
\text { logistic OR quality OR instandhaltung OR wartung OR logistik } \\
\text { OR education OR schulung) AND ( study OR studie OR "Use- } \\
\text { Case" OR application OR anwendung OR system )) AND } \\
\text { ( LIMIT-TO ( PUBYEAR, 2020) OR LIMIT-TO (PUBYEAR, 2019) OR } \\
\text { LIMIT-TO ( PUBYEAR, 2018) OR LIMIT-TO (PUBYEAR, 2017) OR } \\
\text { LIMIT-TO ( PUBYEAR, 2016)) }\end{array}$ & 291 \\
\hline TEMA & $\begin{array}{l}\text { Titel:("Augment* Realit*" OR "AR" OR "Erweitert* Realität") AND (In- } \\
\text { dustr* OR Mangement OR Service OR Maintenance OR Logisic OR } \\
\text { Quality OR Instandhaltung OR Wartung OR Logistik OR Education } \\
\text { OR Schulung) AND (Study OR Studie OR "Use-Case" or Application } \\
\text { or Anwendung or System); Erscheinungsjahr:von } 2016 \text { bis } 2020\end{array}$ & 45 \\
\hline IEEE Explore & $\begin{array}{l}\text { ((("Document Title":"Augmented Reality") OR ("Document Ti- } \\
\text { tle":"AR") OR ("Document Title":"Erweitert* Realität")) AND ( ("Docu- } \\
\text { ment Title":"Industry") OR ("Document Title":"Management") OR } \\
\text { ("Document Title":"Logistic*") OR ("Document Title":Service) OR } \\
\text { ("Document Title":"Quality") OR ("Document Title":"Instandhaltung") } \\
\text { OR ("Document Title":"Wartung") OR ("Document Title":"Logistik") } \\
\text { OR ("Document Title":"Education") OR ("Document Ti- } \\
\text { tle":"Schulung")) AND (("Document Title":"Study") OR ("Document } \\
\text { Title":"Use-Case") OR ("Document Title":"Application") OR ("Docu- } \\
\text { ment Title":"System") OR ("Document Title":"Anwendung") OR } \\
\text { ("Document Title":"Studie"))) }\end{array}$ & 41 \\
\hline \multicolumn{2}{|l|}{ Gesamt } & 563 \\
\hline \multicolumn{2}{|l|}{ Ohne Duplikate } & 283 \\
\hline \multicolumn{2}{|c|}{ Davon relevant auf Basis von Titel und Abstract } & 86 \\
\hline \multicolumn{2}{|c|}{ Davon relevant auf Basis des gesamten Beitrages } & 54 \\
\hline
\end{tabular}


Auf Basis des Titels und der Kurzfassung sind dann Ergebnisse ausgeschlossen, die offensichtlich nicht den Kriterien entsprechen. Dazu gehören Publikationen in anderen Sprachen als Deutsch und Englisch, Publikationen, die keine konkrete Implementierung vorstellen, Systeme ohne industrielle Anwendung und Systeme, die kein AR nutzen. Dabei wird die Definition von AR nach Azuma genutzt. Diese schließt einige als AR bezeichnete Lösungen aus. Insbesondere in den Bereichen Remote Maintenance werden Datenbrille eingesetzt, die einem Arbeiter vor Ort mit einem Experten verbindet. Wenn dabei nur Video-Signale übertragen werden, handelt es sich trotz Einsatz einer Datenbrille noch nicht um AR nach Azuma. Nach Sichtung Kurzfassung wurden 195 Beiträge ausgeschlossen.

Im Anschluss werden die Volltexte heruntergeladen und in das vorgeschlagene System sortiert. Dabei sind 32 weitere Titel auf Grund der oben genannten Kriterien zusätzlich ausgeschlossen worden, so dass insgesamt 54 Titel kategorisiert wurden. Abschließend wurden alle Probleme herausgearbeitet, die auf bei der Umsetzung von AR aufgetreten sind (Ababsa, 2020 - Zhao et al., 2019).

\section{Ergebnisse und Diskussion}

Die Anzahl der Publikationen in den drei Kategorien Aktion, Produktlebenszyklus und dem Grad der Hilfestellung zeigt Abbildung 1: Anzahl der Beiträge in den . Der Großteil der Anwendungen unterstützen bei der Ausführung, z.B. bei Wartungs- und Serviceaufgaben (Morillo et al., 2020) (25 Publikationen) und dem Informieren, z.B. durch die Visualisierung von Betriebsdaten (Baron \& Braune, 2016), (18 Publikationen), das Kontrollieren, z.B. in der Qualitätssicherung (Szajna et al., 2020) (8 Publikationen) und das Planen, z.B. in der Roboterbahnsteuerung (Ong et al., 2020) (3 Publikationen) spielt in der aktuellen Forschung eine eher untergeordnete Rolle.

Über die Hälfte der Systeme unterstützt in sehr hohem Maße (29 Publikationen), wie z. B. Gao et al. (2020), deren System neben detaillierten Beschreibungen auch interaktive 3D Animationen der betroffenen Bauteile zeigt. Durch eine solche Unterstützung im gesamten Prozess zielt das System weniger auf Experten, sondern erlaubt es auch ungelernten Arbeitskräften, den Prozess auszuführen. Ein Beispiel für eine geringe Unterstützung (insgesamt 14 Publikationen) zeigen Coscetti et al. (2020), bei denen sich das System auf die reine Darstellung von Betriebsdaten beschränkt, die dann von Experten interpretiert werden können. Ein System mit mittlerer Hilfestellung (insgesamt 10 Publikationen) zeigt Saggiomo et al. (2016). Das System überlässt wesentlich 
Schritte dem Anwender, unterstützt und führt aber dennoch durch einen strukturierten Prozess, indem es kurze Beschreibungen und eine Orientierungshilfe zeigtAbbildung 1.

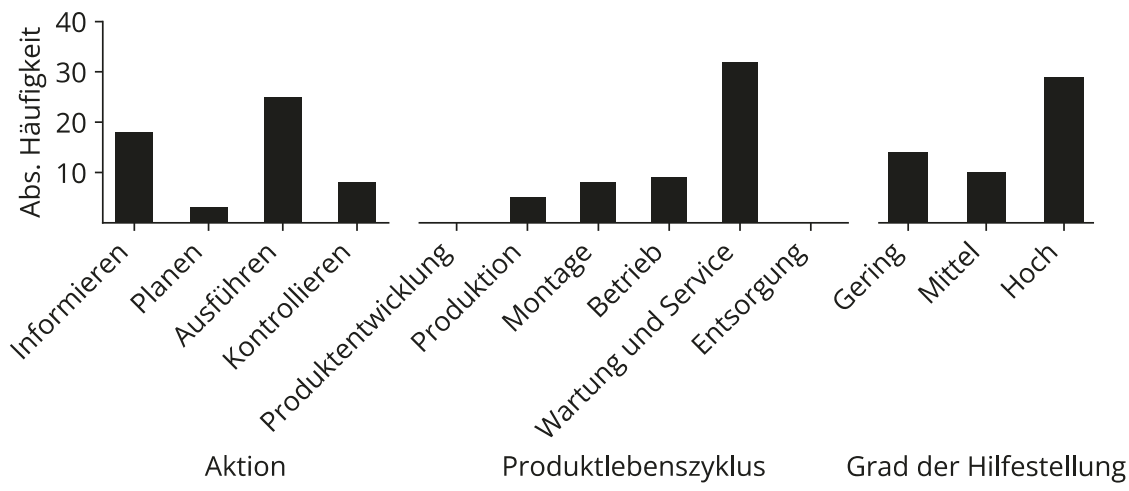

Abbildung 1: Anzahl der Beiträge in den Dimensionen Aktion, Produktlebenszyklus und Grad der Hilfestellung

Abbildung 2 zeigt den Zusammenhang zwischen dem Grad der Hilfestellung und der zu unterstützenden Aktion. Während Systeme, die die Aktionen Planen, Informieren und Kontrollieren unterstützen, gleichmäßig alle drei Hilfestellungsgraden nutzt, setzt die überwiegende Mehrheit der Systeme, die eine konkrete Ausführung unterstützen, auf einen hohen Grad der Hilfestellung. Geringe und mittlere Hilfestellungen, die den Anwender nur durch einen strukturierten Prozess führen, spielen fast keine Rolle. Fast immer wird dem Anwender bei jedem auszuführenden Schritt eine detaillierte, AR gestützte Handlungsanweisung angezeigt. So unterstützen Amza et al. (2018) den Anwender, in dem in jedem Arbeitsschritt animierte Modelle angezeigt werden. Im Hinblick auf den Produktlebenszyklus zeigt sich, dass keine Systeme in den frühen oder späten Phasen (Produktentwicklung, Entsorgung) angewendet werden. Hier fokussiert sich die Forschung eindeutig auf den Betrieb (9 Publikationen) und vor allem Wartung und Service (32 Publikationen). Auch die Produktion (5) und Montage (8) spielen nur eine geringe Rolle.

Neben den drei Dimensionen die vorgeschlagenen Klassifizierungen wurden auch eine Reihe Implementierungsdetails untersucht. In der Kategorie Darstellungstyp wird zwischen der Nutzung von mobilen Smart Devices (30 Publikationen), Head-Mounted-Displays (23 Publikationen) und projektionsbasierten Anwendungen (6 Publikationen) unterschieden. Dies ist die einzige Kategorie, in denen Publikationen in mehrfach gezählt 
wurden, wenn das System z.B. sowohl auf einem mobilen Endgerät als auch auf einem HMD umgesetzt wurde.

In der Kategorie Detailierungsgrad wird die Art des visualisierten Inhaltes unterschieden. Die Mehrzahl der Systeme (29) beschränken sich auf primitive Formen oder Pfeile. 14 Systeme zeigen stark vereinfachte, abstrahierte Geometrien, 11 nutzen detaillierte CAD-Modelle. Kein System versucht, realitätsnahe Inhalte darzustellen, z.B. durch Schattierungen oder Spiegelungen. Zusätzlich wurde der Reifegrad der Systeme untersucht. 34 Publikationen befinden sich in einem frühen Prototypenstatus. 18 Systeme wurden im Rahmen einer Laborstudie validiert, 2 auch in Praxisstudien unter realen Bedingungen. Keine Veröffentlichung beschreibt ein Produktivsystem.

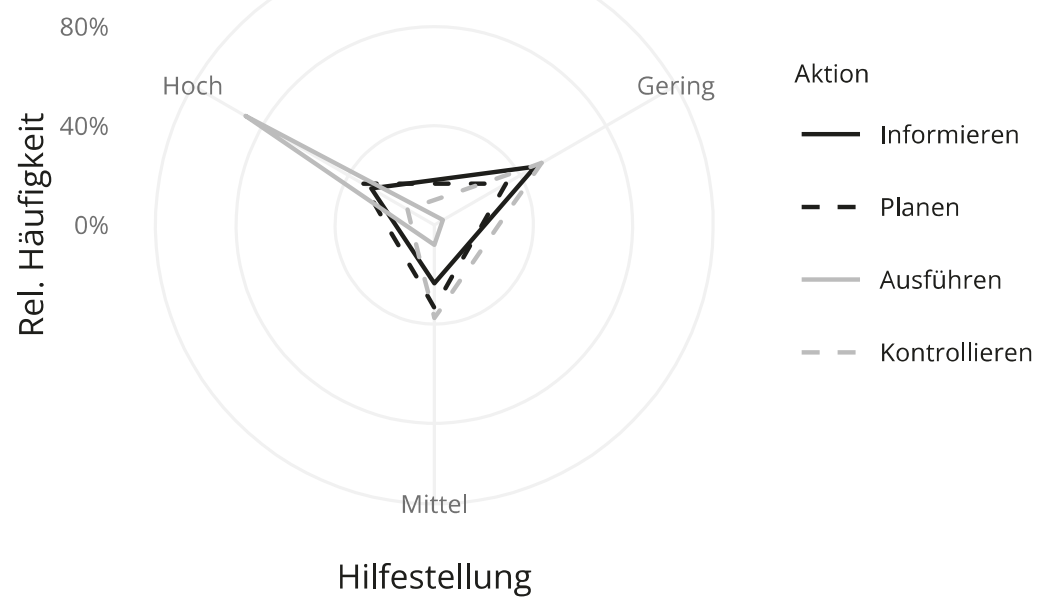

Abbildung 2: Verwendeter Grad der Hilfestellung nach zu unterstützender Aktion

In der letzten untersuchten Kategorie, der Art der Inhaltserstellung, zeigt sich, das mit acht Systemen nur wenige Ansätze vorgestellt werden, in denen die Inhalte möglichst voll automatisiert aus existierenden Daten abgeleitet werden. Zumindest zwölf Systeme versuchen, den Anwender bei der Inhaltserstellung zu unterstützen, z.B. bei Tatic (2017), bei denen durchzuführende Arbeitsschritte strukturiert auf einem zentralen Server abgelegt werden. Bei den übrigen Systemen wird in der Regel keine konkrete Aussage bezüglich der Inhaltserstellung gemacht, so dass von hier von einem statischen Vorgehen für den speziellen Anwendungsfall ausgegangen werden kann. 
Dies ist in Abbildung 3 dargestellt. Anders als z.B. von Azuma angenommen spielen Montageprozesse eher eine untergeordnete Rolle für AR. Ein Grund dafür liegt in den Problemen bei der Umsetzung. So sind die häufig eingesetzten, markerbasierten Lösungen für die Annotation von bei der Montage bewegten Teilen ungeeignet (Vilacoba et al., 2016). Dabei sollten HMDs eingesetzt werden, da dann im Gegensatz zu Tabletbasierten Methoden die Hände des Arbeiters frei bleiben (Vilacoba et al., 2016). Außerdem bieten nicht alle Systeme die für Montageprozesse geforderte Genauigkeit im Tracking (Ababsa, 2020; Amici et al., 2019; Vilacoba et al., 2016). Nachteile von heutigen HMDs sind der Tragekomfort und wenig intuitive Benutzungsschnittstellen (Vilacoba et al., 2016). Dabei sind Tablet-basierte Lösungen im Vorteil, zumal über diese die Einbindung von 2D-Informationen, wie technischen Zeichnungen, einfacher ist (VidalBalea et al., 2020). Ein weiteres Problem bei der Umsetzung von AR-Anwendung ist die Erstellung der Inhalte, die häufig Programmiererfahrungen voraussetzt (Bosch et al., 2020; Mizutani et al., 2017). Hier werden einfach zu bedienende Autorentools gefordert (Akbarinasaji \& Homayounvala, 2017). Der Erstellungsaufwand ist auch eine der Gründe für die häufig hohen Kosten solcher Systeme (Bosch et al., 2020). Zuletzt wird gefordert, das AR-Anwendungen besser auf die Anforderungen, Fähigkeiten und Erwartungen der Endanwender zugeschnitten werden (Bosch et al., 2020).

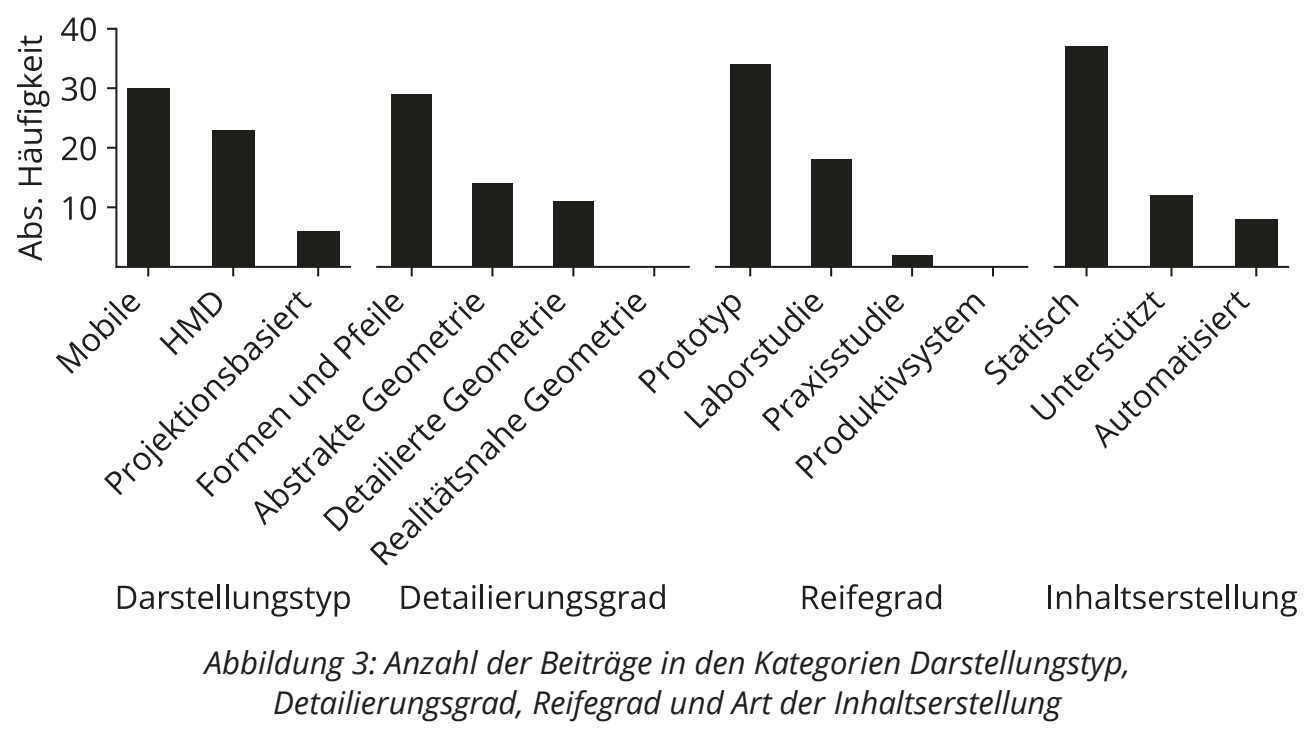




\section{Fazit}

Dieser Beitrag stellt ein Klassifizierungs-Schema für die verschiedenen Einsatzszenarien von AR in der Industrie vor. Es wird gezeigt, dass AR durch die drei Dimensionen Aktion, Lebenszyklusphase und Grad der Hilfestellung charakterisiert werden kann. Dieser Beitrag analysiert vorgestellte Studien und Systeme in einem 5 Jahres-Zeitraum. Bereits bestehende kommerzielle Systeme werden nicht betrachtet. Durch die verwendete Definition von AR nach Azuma werden außerdem einige Systeme des RemoteService nicht betrachtet, die in der Regel einen höheren Reifegrad ausweisen.

Bei heutigen Systemen lassen sich zwei Kategorien von Problemen identifizieren. Technologische Probleme sind dabei Ungenauigkeiten im Tracking, unbequeme und wenig intuitive Hardware, Probleme markerbasierter Lösung und wenig robuste markerlose Trackingsysteme. Es ist zu erwarten, dass diese mit dem technischen Fortschritt durch die Anwender von AR Hardware und SDKs mit der Zeit weniger relevant werden. Mit einer weiteren Verbreitung von AR HMDs im privaten Bereich sinken auch Akzeptanzprobleme bei Mitarbeitern. Daneben gibt es aber auch eine Reihe prozessbezogener Probleme. Zum einen ist die Erstellung von AR Inhalten immer noch zu aufwändig und setzt häufig Programmiererfahrungen voraus, was zu hohen Kosten und einer geringen Anpassungsfähigkeit der Systeme führt. Hier müssen weitere und bessere Autorenwerkzeuge entwickelt werden, die idealerweise bereits auf vorhandene Daten zugreifen. Außerdem müssen die Bedürfnisse der Zielgruppe besser berücksichtigt werden. So braucht ein Facharbeiter andere Informationen als eine ungelernte Arbeitskraft. Dennoch versuchen die meisten Systeme, durchgehend eine hohe Unterstützung anzubieten, bei der z.B. jede Schraube markiert und zum Teil auch durch 3D-Modelle und Animationen dargestellt werden. Hier sollten die Anforderungen der Zielgruppe beim Design der Systeme kritisch hinterfragt werden.

\section{Literaturverzeichnis}

Azuma, R. T. (1997). A survey of augmented reality. Presence: Teleoperators and Virtual Environments, 6(4), 355-385.

Caudell, T. P. \& Mizell, D. W. (1992). Augmented Reality: An Application of Heads-Up Display Technology to Manual Manufacturing Processes. In Hawaii international conference on system sciences (S. 659-669).

Del Fernández Amo, I., Erkoyuncu, J. A., Roy, R., Palmarini, R. \& Onoufriou, D. (2018). A systematic review of Augmented Reality content-related techniques for knowledge transfer in maintenance applications. Computers in Industry, 103, 47-71.

Fraga-Lamas, P., Fernandez-Carames, T. M., Blanco-Novoa, 0. \& Vilar-Montesinos, M. A. (2018). A Review on Industrial Augmented Reality Systems for the Industry 4.0 Shipyard. IEEE Access, 6, 13358-13375. 
Bottani, E. \& Vignali, G. (2019). Augmented reality technology in the manufacturing industry: A review of the last decade. IISE Transactions, 51(3), 284-310.

Souza Cardoso, L. F. de, Mariano, Flávia Cristina Martins Queiroz \& Zorzal, E. R. (2019). A Survey of Industrial Augmented Reality. Computers \& Industrial Engineering, 139, 106159.

Egger, J. \& Masood, T. (2020). Augmented reality in support of intelligent manufacturing - A systematic literature review. Computers \& Industrial Engineering, 140, 106195.

Normand, J.- M., Servières, M. \& Moreau, G. (2012). A new typology of augmented reality applications. Proceedings of the 3rd Augmented Human International Conference on - $A H^{\prime} 12$.

Brockmann, T., Krueger, N., Stieglitz, S. \& Bohlsen, I. (2013). A framework for collaborative augmented reality applications. In Proceedings of the Nineteenth Americas Conference on Information Systems. Chicago, United States.

Wang, X., Kim, M. J., Love, P. E. \& Kang, S.- C. (2013). Augmented Reality in built environment: Classification and implications for future research. Automation in Construction, 32, 1-13.

Fite-Georgel, P. (2011). Is there a reality in Industrial Augmented Reality? 2011 10th IEEE International Symposium on Mixed and Augmented Reality.

Ludwig, B. (2015). Planbasierte Mensch-Maschine-Interaktion in multimodalen Assistenzsystemen. Berlin, Heidelberg: Springer Berlin Heidelberg. https://doi.org/10.1007/978-3-662-44819-9

Röltgen, D. \& Dumitrescu, R. (2020). Classification of industrial Augmented Reality use cases. Procedia CIRP, (91), 93-100.

Grieves, M. (2006). Product lifecycle management. Driving the next generation of lean thinking; [ [how GE, P\&G, Ford, Toyota, an other leading companies achieved dramatic increases in productivity and profit. New York: McGraw-Hill. Retrieved from http://www.loc.gov/catdir/enhancements/fy0668/2006280824-b.html

Erkoyuncu, J. A., Del Amo, I. F., Dalle Mura, M., Roy, R. \& Dini, G. (2017). Improving efficiency of industrial maintenance with context aware adaptive authoring in augmented reality. CIRP Annals, 66(1), 465-468.

Gattullo, M., Evangelista, A., Manghisi, V. M., Uva, A. E. [Antonio E.], Fiorentino, M., Boccaccio, A. et al. (2020). Towards Next Generation Technical Documentation in Augmented Reality Using a Context-Aware Information Manager. Applied Sciences, 10(3), 780.

Tranfield, D., Denyer, D. \& Smart, P. (2003). Towards a Methodology for Developing Evidence-Informed Management Knowledge by Means of Systematic Review. British Journal of Management, 14, 207-222.

Ababsa, F. (2020). Augmented Reality Application in Manufacturing Industry: Maintenance and Non-destructive Testing (NDT) Use Cases, 12243, 333-344. https://doi.org/10.1007/978-3-030-58468-9_24

Akbarinasaji, S. \& Homayounvala, E. (2017). A novel context-aware augmented reality framework for maintenance systems. Journal of Ambient Intelligence and Smart Environments, 9(3), 315-327. https://doi.org/10.3233/AlS-170435

Amici, T. T., Filho, P. H. \& Campo, A. B. (2019). Augmented Reality Applied to a Wireless Power Measurement System of an Industrial 4.0 Advanced Manufacturing Line, 1402-1406. https://doi.org/10.1109/INDUSCON.2018.8627301

Amza, C. G., Zapciu, A. \& Teodorescu, 0. (2018). Augmented reality application for industrial non-destructive inspection training, 30002. https://doi.org/10.1063/1.5024152 
Ariansyah, D., Rosa, F. \& Colombo, G. (2019). Smart Maintenance: A Wearable Augmented Reality Application Integrated with CMMS to Minimize Unscheduled Downtime. Computer-Aided Design and Applications, 17(4), 740-751.

https://doi.org/10.14733/cadaps.2020.740-751

Aschenbrenner, D., Rojkov, M., Leutert, F., Verlinden, J., Lukosch, S., Latoschik, M. E. et al. (2018). Comparing Different Augmented Reality Support Applications for Cooperative Repair of an Industrial Robot, 69-74. https://doi.org/10.1109/ISMAR-Adjunct.2018.00036

Aschenbrenner, D., Latoschik, M. E. \& Schilling, K. (2016). Industrial maintenance with augmented reality, 341-342. https://doi.org/10.1145/2993369.2996305

Avalle, G., Pace, F. de, Fornaro, C., Manuri, F. \& Sanna, A. (2019). An Augmented Reality System to Support Fault Visualization in Industrial Robotic Tasks. IEEE Access, 7, 132343-132359. https://doi.org/10.1109/ACCESS.2019.2940887

Baron, L. \& Braune, A. (2016). Case study on applying augmented reality for process supervision in industrial use cases, 14. https://doi.org/10.1109/ETFA.2016.7733639

Bellalouna, F. (2020). Industrial Use Cases for Augmented Reality Application, 11-18. https://doi.org/10.1109/Cog|nfoCom50765.2020.9237882

Bosch, T., van Rhijn, G., Krause, F., Könemann, R., Wilschut, E. S. \& Looze, M. de. (2020). Spatial augmented reality, 1-7. https://doi.org/10.1145/3389189.3397975

Brice, D., Rafferty, K. \& McLoone, S. (2020). AugmenTech: The Usability Evaluation of an AR System for Maintenance in Industry, 12243, 284-303. https://doi.org/10.1007/978-3-030-58468-9_21

Büttner, S., Prilla, M. \& Röcker, C. (2020). Augmented Reality Training for Industrial Assembly Work - Are Projection-based AR Assistive Systems an Appropriate Tool for Assembly Training?, 1-12. https://doi.org/10.1145/3313831.3376720

Coscetti, S., Moroni, D., Pieri, G. \& Tampucci, M. (2020). Factory Maintenance Application Using Augmented Reality, 1-6. https://doi.org/10.1145/3378184.3378218

Mourtzis, D. [D.], Zogopoulos, V. [V.] \& Vlachou, E. [E.]. (2017). Augmented Reality Application to Support Remote Maintenance as a Service in the Robotics Industry. Procedia CIRP, 63, 46-51. https://doi.org/10.1016/j.procir.2017.03.154

Damiani, L., Revetria, R. \& Morra, E. (2020). Safety in Industry 4.0: The Multi-Purpose Applications of Augmented Reality in Digital Factories. Advances in Science, Technology and Engineering Systems Journal, 5(2), 248-253.

https://doi.org/10.25046/aj050232

Di Pace, A., Fenza, G., Gallo, M., Loia, V., Meglio, A. \& Orciuoli, F. (2020). Implementing the Cognition Level for Industry 4.0 by Integrating Augmented Reality and Manufacturing Execution Systems, 1151, 957-967. https://doi.org/10.1007/978-3030-44041-1_83

Mourtzis, D. [Dimitris], Samothrakis, V., Zogopoulos, V. [Vasilios] \& Vlachou, E. [Ekaterini]. (2019). Warehouse Design and Operation using Augmented Reality technology: A Papermaking Industry Case Study. Procedia CIRP, 79, 574-579.

https://doi.org/10.1016/j.procir.2019.02.097

Mourtzis, D. [Dimitris], Vlachou, A. \& Zogopoulos, V. [Vasilios]. (2017). Cloud-Based Augmented Reality Remote Maintenance Through Shop-Floor Monitoring: A Product-Service System Approach. Journal of Manufacturing Science and Engineering, 139(6). https://doi.org/10.1115/1.4035721 
Mourtzis, D. [Dimitris], Zogopoulos, V. [Vasilios], Katagis, I. \& Lagios, P. (2018). Augmented Reality based Visualization of CAM Instructions towards Industry 4.0 paradigm: a CNC Bending Machine case study. Procedia CIRP, 70, 368-373. https://doi.org/10.1016/j.procir.2018.02.045

Erdei, T., Molnár, Z., Obinna, N. C. \& Husi, G. (2018). A Novel Design of an Augmented Reality Based Navigation System \& its Industrial Applications. ACTA IMEKO, 7(1), 57. https://doi.org/10.21014/acta_imeko.v7i1.528

Fiorentino, M., Radkowski, R., Boccaccio, A. \& Uva, A. E. [Antonio Emmanuele]. (2016). Magic Mirror Interface for Augmented Reality Maintenance, 160-167. https://doi.org/10.1145/2909132.2909268

Gao, L., Wu, F., Liu, L. \& Wan, X. (2020). Construction of Equipment Maintenance Guiding System and Research on Key Technologies Based on Augmented Reality, 634, 275-282. https://doi.org/10.1007/978-981-15-2341-0_34

Gomes Jr, D. L., Reis, P. R. J. d., Paiva, A. C. de, Silva, A. C., Braz Jr, G., Gattass, M. et al. (2017). An Approach for Construction of Augmented Reality Systems using Natural Markers and Mobile Sensors in Industrial Fields. International Journal of Computers Communications \& Control, 12(4), 507. https://doi.org/10.15837/ijccc.2017.4.2658

Gong, Y., Su, C. \& Wei, Y. (2019). An Interactive Task Modeling Method for the Development of Augmented Reality Maintenance Guiding System, 136-140. https://doi.org/10.1109//HMSC.2019.00039

Guler, 0. \& Yucedag, I. (2018). Developing an CNC lathe augmented reality application for industrial maintanance training, 1-6. https://doi.org/10.1109/ISMSIT.2018.8567255

Hamidane, R., MOUSS, L. H., Bellarbi, A. \& MAHDAOUI, R. (2018). Implementation of a Preventive Maintenance System Based on Augmented Reality, 1-6. https://doi.org/10.1109/PAIS.2018.8598510

Haramaki, T. \& Nishino, H. (2016). An Engineering Education Support System Using Projection-Based AR, $267-272$. https://doi.org/10.1109/NBiS.2016.67

Knopp, S., Klimant, P. \& Allmacher, C. (2019). Industrial Use Case - AR Guidance using Hololens for Assembly and Disassembly of a Modular Mold, with Live Streaming for Collaborative Support, 134-135. https://doi.org/10.1109/ISMAR-Adjunct.2019.00-63

Kollatsch, C., Schumann, M., Klimant, P. \& Lorenz, M. (2017) [POSTER] Industrial Augmented Reality: Transferring a Numerical Control Connected Augmented Realty System from Marketing to Maintenance, 39-41. https://doi.org/10.1109/ISMARAdjunct.2017.27

Koteleva, N., Buslaev, G., Valnev, V. \& Kunshin, A. (2020). Augmented Reality System and Maintenance of Oil Pumps. International Journal of Engineering, 33(8). https://doi.org/10.5829/ije.2020.33.08b.20

Lampen, E., Lehwald, J. \& Pfeiffer, T. (2020). Virtual Humans in AR: Evaluation of Presentation Concepts in an Industrial Assistance Use Case, 1-5. https://doi.org/10.1145/3385956.3418974

Li, L., Xing-zhi, W. \& Yao-giang, X. (2016). Augmented Reality in IT Operation Management of Power System, 12-17. https://doi.org/10.1109/WISA.2016.13

Manuri, F., Pizzigalli, A. \& Sanna, A. (2019). A State Validation System for Augmented Reality Based Maintenance Procedures. Applied Sciences, 9(10), 2115. https://doi.org/10.3390/app9102115

Mizutani, I., Kritzler, M., Garcia, K. \& Michahelles, F. (2017). Intuitive interaction with semantics using augmented reality, 1-7. https://doi.org/10.1145/3131542.3131550 
Molina, J. C., Chiliquinga, G., Saeteros, M. \& Caiza, G. (2020). Real-Time Augmented Reality Application for Visualizing Variables in Industrial Processes, 1307, 348-362. https://doi.org/10.1007/978-3-030-62833-8_26

Morillo, P., García-García, I., Orduña, J. M., Fernández, M. \& Juan, M. C. (2020). Comparative study of AR versus video tutorials for minor maintenance operations. Multimedia Tools and Applications, 79(11-12), 7073-7100.

https://doi.org/10.1007/s11042-019-08437-9

Nithyanandam, G. K., Kothandaraman, P. K., Munguia, J. \& Marimuthu, M. (2020). Implementing Marked-Based Augmented Reality in Discrete industry - A Case Study, 1-6. https://doi.org/10.1109/HYDCON48903.2020.9242696

Ong, S. K., Yew, A., Thanigaivel, N. K. \& Nee, A. (2020). Augmented reality-assisted robot programming system for industrial applications. Robotics and Computer-Integrated Manufacturing, 61, 101820. https://doi.org/10.1016/j.rcim.2019.101820

Peng, Y., Yu, G., Ni, W., LV, Z., Jiang, Y. \& Chen, J. (2017). Design and development of intelligent operation and maintenance training system for substation based on augmented reality, 4765-4769. https://doi.org/10.1109/CAC.2017.8243621

Pierdicca, R., Frontoni, E., Pollini, R., Trani, M. \& Verdini, L. (2017). The Use of Augmented Reality Glasses for the Application in Industry 4.0, 10324, 389-401. https://doi.org/10.1007/978-3-319-60922-5_30

Pringle, A., Hutka, S., Mom, J., van Esch, R., Heffernan, N. \& Chen, P. (2019). Ethnographic study of a commercially available augmented reality HMD app for industry work instruction, 389-397. https://doi.org/10.1145/3316782.3322752

Qian, K., Zhang, X., Yu, H. \& Ma, X. (2018). Industrial Maintenance and Assembly Guidance Using a Markerless AR System with Monocular Camera, 1911-1916. https://doi.org/10.1109/ICARCV.2018.8581087

Quandt, M., Beinke, T. \& Freitag, M. (2020). User-Centered Evaluation of an Augmented Reality-based Assistance System for Maintenance. Procedia CIRP, 93, 921-926. https://doi.org/10.1016/j.procir.2020.03.053

Saggiomo, M., Loehrer, M., Kerpen, D., Lemm, J. \& Gloy, Y.- S. (2016). Human-and Task-Centered Assistance Systems in Production Processes of the Textile Industry: Determination of Operator-Critical Weaving Machine Components for AR-Prototype Development, 560-568. https://doi.org/10.1109/HICSS.2016.76

Siew, C. Y., Nee, A. Y. C. \& Ong, S. K. (2019). Improving Maintenance Efficiency with an Adaptive AR-assisted Maintenance System, 74-78. https://doi.org/10.1145/3351180.3351203

Siew, C. Y., Ong, S. K. \& Nee, A. (2019). A practical augmented reality-assisted maintenance system framework for adaptive user support. Robotics and Computer-Integrated Manufacturing, 59, 115-129. https://doi.org/10.1016/j.rcim.2019.03.010

Su, Y. H., Liao, C. F., Ko, C. H., Cheng, S. L. \& Young, K. Y. (2018). An AR-Based Manipulation System for Industrial Robots, 1282-1285. https://doi.org/10.1109/ASCC.2017.8287355

Szajna, A., Stryjski, R., Woźniak, W., Chamier-Gliszczyński, N. \& Królikowski, T. (2020). The Production Quality Control Process, Enhanced with Augmented Reality Glasses and the New Generation Computing Support System. Procedia Computer Science, 176, 3618-3625. https://doi.org/10.1016/j.procs.2020.09.024

Tatic, D. (2018). An augmented reality system for improving health and safety in the electro-energetics industry. Facta universitatis - series: Electronics and Energetics, 31(4), 585-598. https://doi.org/10.2298/FUEE1804585T

Vidal-Balea, A., Blanco-Novoa, O., Fraga-Lamas, P., Vilar-Montesinos, M. \& Fernández-Caramés, T. M. (2020). Creating Collaborative Augmented Reality Experiences for Industry 4.0 Training and Assistance Applications: Performance Evaluation in the Shipyard of the Future. Applied Sciences, 10(24), 9073. https://doi.org/10.3390/app10249073 
Vilacoba, D., Trujillo, M. A., Vinuales, A. \& Weber, P. (2016). Press dedicated machine Show-room, a direct application of Augmented reality in industry. An industrial Augmented reality experience, 1990-1995.

https://doi.org/10.1109/ICIT.2016.7475072

Vogel, C., Schulenburg, E. \& Elkmann, N. (2020). Projective- AR Assistance System for shared Human-Robot Workplaces in Industrial Applications, 1259-1262. https://doi.org/10.1109/ETFA46521.2020.9211953

Zhao, P., Wu, H., Shi, X., Li, J., Yi, X. \& Liu, S. (2019). Research on maintenance guiding system based on augmented reality, 944-949. https://doi.org/10.1109/SDPC.2019.00180

\section{Kontakt}

Jan Luca Siewert, M. Sc.

Dr.-Ing. Matthias Neges

Univ.-Prof. Dr.-Ing. Detlef Gerhard

Ruhr-Universität Bochum

Universitätsstr. 150

44801 Bochum

www.lde.rub.de 